\title{
Intrinsic differences in sensitivity to 5-HT between high- and low-output terminals innervating the same target
}

\author{
Robin L. Cooper*, Ahmet Dönmezer, Joseph Shearer \\ Department of Biology, University of Kentucky, Lexington, KY 40506-0225, USA
}

Received 17 June 2002; accepted 18 October 2002

\begin{abstract}
The differential action of neuromodulators on synapses of various efficacy provides additional fine tuning of synaptic regulation beyond frequency induced plasticity. We used the well-characterized high- and low-output motor nerve terminals, of the tonic and phasic neuromuscular junctions (NMJs) in the walking leg extensor muscle of the crayfish, to investigate differential actions of serotonin (5-HT) since both terminals innervate the same target. The excitatory postsynaptic potentials of the tonic NMJ are enhanced to a greater extent than for the phasic NMJs during exposure to 5-HT (100 nM). Macropatch current recordings at identified sites along the motor nerve terminals and quantal analysis indicate that mean quantal content is substantially increased by 5-HT. The overall probability of vesicular release increases to a greater extent at tonic terminals than at phasic terminals when exposed to $100 \mathrm{nM}$ 5-HT. Measures in the area (i.e. charge) of spontaneous quantal currents indicate no difference in postsynaptic receptivity to the glutamatergic synaptic transmission upon exposure to 5-HT. The results provide new details concerning differential modulation of low- and high-output synapses present on the same target tissue.

(C) 2002 Elsevier Science Ireland Ltd and the Japan Neuroscience Society. All rights reserved.
\end{abstract}

Keywords: Neurotransmission; Crayfish; Neuromuscular junction; Synapse; Serotonin

\section{Introduction}

Differences in synaptic properties among neurons are wide ranging, from regulation in the extent of synaptic contacts, biochemical calcium buffering differences, to differences in the type of calcium channels. In some cases, the synaptic area can be similar between junctions but the amount of synaptic output under the same environmental conditions is markedly different. Endogenous compounds that are released into the circulation, or locally, that alter neuronal activity are referred to as neuromodulators. Depending on the location of the receptors, receptor subtypes and densities, neuromodulators can discretely alter synaptic output. The range in which neuromodulators, such as biogenic amines (i.e. serotonin), modulate synaptic transmission may also depend on the degree of inherent synaptic capacity. If synapses are operating at near maximum output, then a

\footnotetext{
* Corresponding author. Tel.: +1-859-257-5050; fax: +1-859-2571717

E-mail address: rlcoop1@pop.uky.edu (R.L. Cooper).
}

stimulatory synaptic neuromodulator will have less impact in enhancing release than at synapses which are operating further from maximal output. Neuromodulators can also act on postsynaptic targets by altering receptivity to transmission. Thus, a preparation in which both high- and low-output presynaptic terminals innervate the same target holds promise in understanding some of the aspects to account for differential modulation among synaptic sites.

Neuromodulation is influential in the regulation of synaptic transmission within the mammalian and invertebrate central nervous system as well as at peripheral neuromuscular junctions (NMJs) of invertebrates (Kupfermann, 1979). Depending on the modulator, synaptic transmission may either be enhanced or depressed. Due to the relative simplicity of crustacean NMJs and the ability to measure pre- and postsynaptic components discretely, the mechanistic actions of neuromodulators on synaptic plasticity can readily be differentiated. Depending on the particular crustacean skeletal muscle, individual muscle fibers may be innervated by a single phasic or single tonic motor neuron or 
multiples of both types of motor neurons (Atwood, 1973, 1976; Atwood and Cooper, 1995, 1996a,b; LaFramboise et al., 1999).

The leg extensor muscle in the walking legs of the crayfish Procambarus clarkii is such a preparation in which both phasic and tonic motor neurons innervate the same muscle fibers in close apposition; therefore, the intrinsic differences among the terminals can be studied without regard to differential postsynaptic influences. Although the varicosities of tonic terminals are much larger, they have a significantly lower transmitter output than phasic terminals at low frequencies of nerve impulse activity (Bradacs et al., 1997; King et al., 1996; Msghina et al., 1998). The two excitatory motor neurons are morphologically different. The tonic terminals are very active in freely moving animals, and their synaptic terminals are larger, more varicose and contain a greater proportion of mitochondria than those of phasic axons. The phasic axons are mainly inactive, firing brief bursts during walking, and their synaptic terminals are small, thin and run parallel to those of the tonic axons (Bradacs et al., 1997). The two types of motor nerve terminals are known to have differences in the calcium binding protein frequenin (Jeromin et al., 1999) and the amount of calcium entry (Msghina et al., 1999) and they demonstrate activity-dependent differences in vesicle uptake and release (Quigley et al., 1999).

The graded tonic excitatory postsynaptic potentials (EPSPs) in the extensor muscle are quite small at low frequencies but show pronounced facilitation at high stimulation frequencies. The phasic EPSPs are large at low frequencies and show some facilitation with higher frequencies, but facilitation is not as pronounced as for tonic EPSPs. The investigation of the differential influence of neuromodulators on the phasic and tonic terminals allows assessment of intrinsic differences between the neurons to be assessed. Determining the differential actions of serotonin (5-HT) on this preparation is a suitable starting point since it is endogenous to the crayfish hemolymph and has direct actions on crustacean NMJs.

In crayfish and lobster, 5-HT is released into the hemolymph from nerve endings in the 2 nd thoracic roots and from the pericardial organs (Beltz and Kravitz, 1983). At crayfish NMJs, 5-HT enhances synaptic transmission (Dudel, 1965; Florey and Florey, 1954; Fisher and Florey, 1983). Primarily, 5-HT enhances the probability of vesicular fusion during evoked transmission, but it can also increase the number of vesicular release sites (Southard et al., 2000; Strawn et al., 2000). The cellular mechanism may involve phosphorylation of vesicular docking related synaptic proteins, enhancing the number of vesicles to be released upon an evoked response (He et al., 1999; Cooper et al., 2001; Tabor and Cooper, 2002).
Since the tonic terminals show many more synapses structurally per length of terminal than the phasic counterpart but display a lower synaptic output (King et al., 1996; Bradacs et al., 1997), we initially postulated that the tonic terminals would show a greater effect to the excitatory neuromodulator serotonin $(5-\mathrm{HT})$ than phasic terminals by recruiting silent synaptic sites during neuromodulation. To examine differences in the action of 5-HT on the two types of nerve terminals, measures of EPSPs and excitatory postsynaptic currents (EPSCs), from discrete regions of the terminals, were used for comparisons of altered synaptic function.

This study has previously been reported in abstract form (Shearer et al., 2000).

\section{Methods}

\subsection{Animals and anatomy}

All experiments were performed using the first walking leg of crayfish, Procambarus clarkii, measuring 4-6 $\mathrm{cm}$ in body length (Atchafalaya Biological Supply Co., Raceland, LA). Preparations were dissected according to standard procedures (Bradacs et al., 1997). The tissue was pinned out in a Slygard dish as to expose the muscle in a taut position. The extensor muscle was viewed with a Nikon, Optiphot-2 upright fluorescent microscope using a $40 \times(0.55$ NA) Nikon water immersion objective. Dissected preparations were maintained in crayfish saline, a modified Van Harreveld's solution $\left(205 \mathrm{mM} \mathrm{NaCl} ; 5.3 \mathrm{mM} \mathrm{KCl} ; 13.5 \mathrm{mM} \mathrm{CaCl} \cdot 2 \mathrm{H}_{2} \mathrm{O}\right.$; $2.45 \mathrm{mM} \mathrm{MgCl}{ }_{2} \cdot 6 \mathrm{H}_{2} \mathrm{O} ; 0.5 \mathrm{mM}$ HEPES adjusted to $\mathrm{pH}$ 7.4) at $14{ }^{\circ} \mathrm{C}$. The entire extensor muscle is innervated by a single tonic and a single phasic excitatory motor neuron (Bradacs et al., 1997). In saline containing 50\% reduced calcium, the concentration used was $6.75 \mathrm{mM}$ $\mathrm{CaCl}_{2} \cdot 2 \mathrm{H}_{2} \mathrm{O}$.

\subsection{Physiology}

\subsubsection{Evoked postsynaptic potentials}

Intracellular muscle recordings were made with a $3 \mathrm{M}$ $\mathrm{KCl}$-containing microelectrode. The short-term facilitation (STF) was provided by giving a $50 \mathrm{~Hz}$ train of ten pulses at intervals of $10 \mathrm{~s}$. To determine a facilitation index (FI) for the induced STF of the tonic EPSPs, the amplitude of the 10th, 15th, or 20th EPSP within a train is compared with one of the preceding EPSPs amplitude within the response (Crider and Cooper, 2000). The subtraction of the numeral one ensures that if there is no facilitation occurring, the FI will then be zero. When recording EPSPs, as a result of stimulating the phasic motor neuron, a stimulus rate of $0.5 \mathrm{~Hz}$ was used in order not to promote synaptic depression. 
Responses were recorded as described earlier (Crider and Cooper, 1999). Selective stimulation of the phasic or tonic excitatory axons was carried out by using a 'macropatch' electrode with an inner diameter of 15$20 \mu \mathrm{m}$ placed directly on either the tonic or phasic axon (Bradacs et al., 1997). The axon type is easily identified after staining with the vital dye 4-Di-2-ASP since the tonic axon is brighter, due to the larger number of mitochondria within it (Atwood and Nguyen, 1995).

\subsubsection{Excitatory postsynaptic currents}

The living terminals were visualized by exposure to vital fluorescent dye 4-Di-2-ASP (Molecular Probes; Cooper et al., 1995a; Magrassi et al., 1987). The synaptic currents were obtained using a loose patch technique. This was done by lightly placing a $10-20 \mu \mathrm{m}$ fire polished glass electrode directly over a spatially isolated varicosity along the visualized nerve terminal. The macropatch electrode is specific for recoding currents within the region of the electrode lumen. The seal resistance was in the range of 100-1 M $\Omega$. The evoked EPSCs and miniature excitatory postsynaptic currents (mEPSCs) were recorded and analyzed to determine the mean quantal content $(m)$ (Cooper et al., 1995b, 1996a). Mean quantal content and the number of failures in evoked release were determined by the direct quantal counting method for the low-output tonic terminals. If only one single event occurred after the spike, it was counted as one; when double events occurred, they were referred to as two, etc... Quantal release over the time was also monitored by examining the area of the evoked current (a measure of charge). The time of the peak in evoked events varies due to latency jitter, so when multiple events occur, the measure of peak amplitude is not as reliable as the area measure (Cooper et al., 1995b). To monitor quanta released over time, the area of the evoked current was measured for each event. This approach was also used to quantify alterations in synaptic currents for the tonic terminals. Since the phasic terminal releases large multiples of vesicles within the macropatch region, the analysis of synaptic charge was used to measure the effects of 5-HT instead of directly counting quantal events. The tonic axon was stimulated at a rate of $1 \mathrm{~Hz}$ in order not to facilitate the responses between trials and the phasic axon was stimulated at $0.5 \mathrm{~Hz}$ to avoid synaptic depression.

Since exposure to 5-HT has a gradual effect on all of the quantal parameters, sample sets of data for every 500 events were used (Table 1). To obtain estimates of the quantal parameters for the probability of vesicular release $(p)$ and the number of release sites $(n)$, data sets were tested for a best-fit approximation based on assumptions discussed in earlier reports (Cooper et al., 1995b; Wojtowicz et al., 1991). Binomial distributions are known to represent the quantal nature of release in crayfish NMJs (Wojtowicz et al., 1991). To test for non-
Table 1

Direct counts of the quantal events recorded from tonic terminals before and during exposure to 5-HT (100 nM)

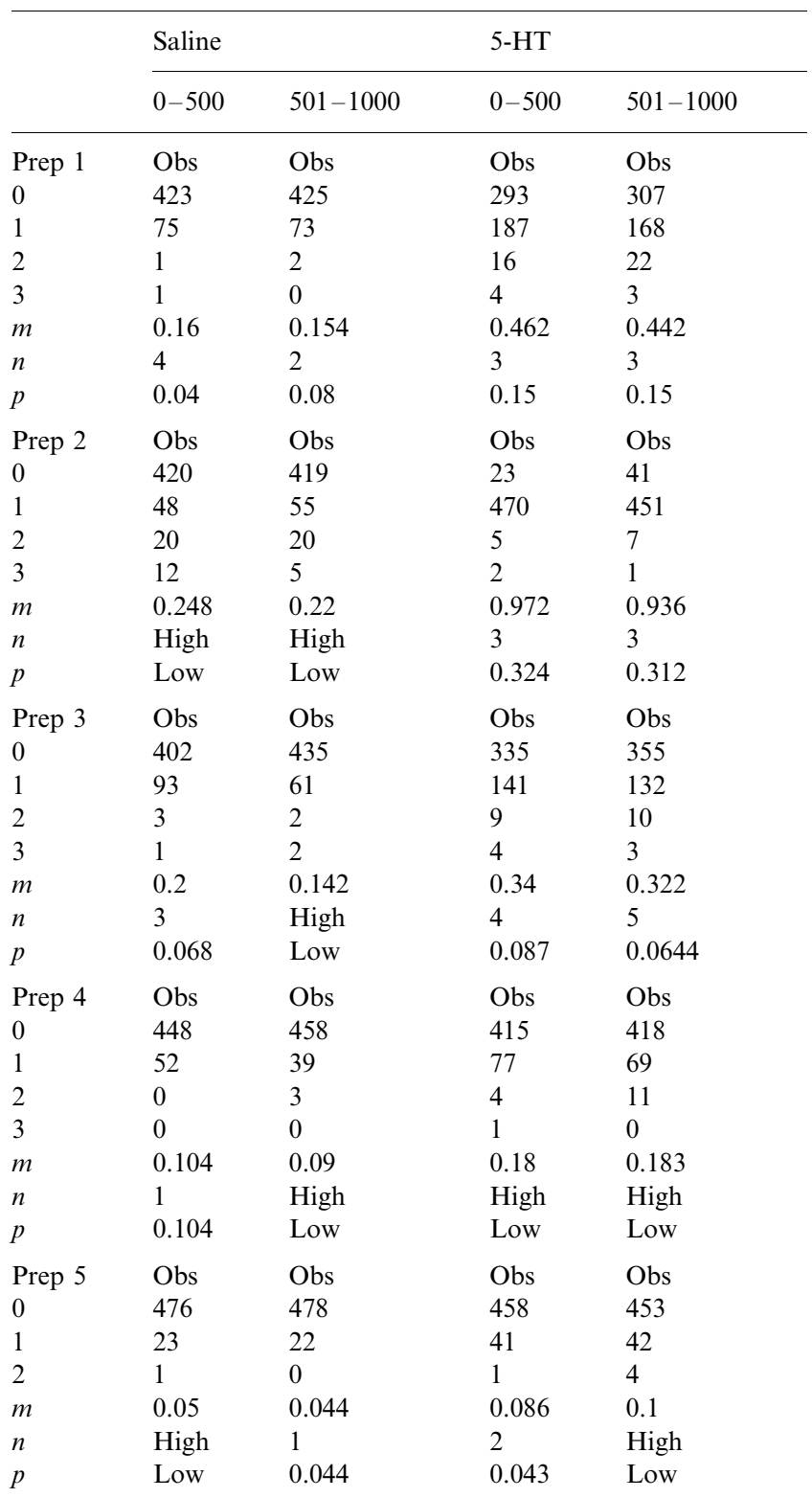

The first column states the number of discrete events ( 0 , failures; 1 , single events; 2 , double events; etc...) that occurred per stimulus trial. The second column states the observed number of occurrences during each of the two sets of 500 trials before and during exposure to 5-HT, respectively. The mean quantal content $(\mathrm{m})$ for each condition is shown for each of the 500 trials. To obtain estimates of the quantal parameters for the probability of vesicular release $(p)$ and the number of release sites $(n)$, data sets were tested for a best-fit approximation.

uniform binomial distributions, the procedures described earlier were used (Wojtowicz et al., 1991). The chi-squared statistic $\left(\chi^{2}\right)$ and a modified Akaike information criterion were used to estimate the distribution that best-fit the observed distribution of events. Further estimates of $n$ and $p$ were obtained by MLE as described in Smith et al. (1991) and a bootstrap algorithm 
(provided by Dr Bruce Smith, Dalhouse University, Halifax, Canada) of 1000 iterations as described by Efron and Tibshirani (1993).

\subsection{Application of neuromodulators}

To apply exogenous compounds to the preparation, the bathing medium was rapidly exchanged with saline containing 5-HT (100 nM, Sigma). Standards of $10 \mu \mathrm{M}$ of 5-HT were made in saline and frozen. A fresh aliquot was used before each experiment.

\subsection{Statistical analysis}

To quantitatively compare the change in the EPSP amplitudes and synaptic charge from the EPSCs, the measurements were normalized to a percent difference. The percent difference was calculated using the difference among the average response during baseline recording prior to 5-HT exposure and the maximum response during the exposure. This difference was then dividing by the baseline value as shown in the following equation:

[(Maximum response-Baseline $) /$ Baseline $] \times 100$

$=\%$ Difference.

When the basic assumption of parametric Student $t$ test was valid it was used; otherwise, the non-parametric Wilcoxon rank sum test was used.

\section{Results}

\subsection{Influence of 5-HT on the amplitude of tonic and phasic EPSPs}

With selective stimulation of the tonic or phasic axon, at the proximal end of the extensor nerve bundle, the corresponding tonic or phasic EPSPs were readily elicited and monitored in muscle fibers with an intracellular electrode (Fig. 1). Since there is variation along the proximal and distal muscle fibers as well as the deep and superficial fibers in the extensor muscle (Bradacs et al., 1997), only the superficial fibers in the middle aspect of the extensor muscle were used in this study. The morphology of the tonic and phasic terminals were very distinct (Fig. 1B). The terminals of the tonic exciter had intermediately spaced varicosities (i.e. swellings of the terminal). Previous work in serial sectioning of these regions had shown that the majority of synapses occur within the varicosities and not in the bottleneck regions (Florey and Cahill, 1982; Cooper et al., 1996a; King et al., 1996; Msghina et al., 1998). From photos, the innervation profiles on the fibers are able to be traced as shown (Fig. 1B). The sizes of the EPSPs evoked by a single stimulus were markedly different. The phasic

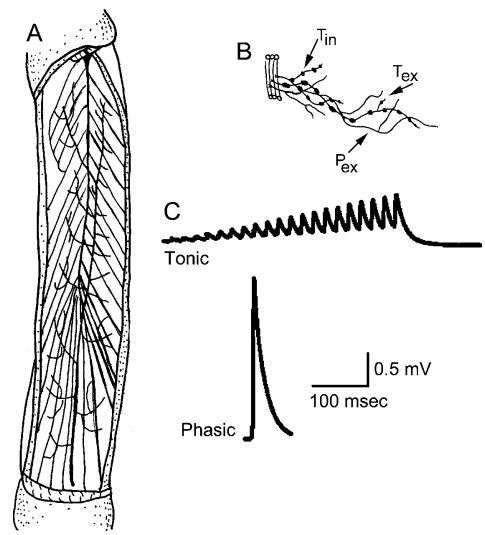

Fig. 1. In the leg extensor neuromuscular preparation, each muscle fiber is innervated by both a phasic and tonic excitatory motor neuron. (A) The medial (or deep) surface of the muscle is readily assessable after removal of the flexor muscle. The distal end of the muscle is at the top. (B) The muscle fibers are innervated by a tonic excitory (Tex) and inhibitor (Tin) with both terminals having intermediate spaced varicosities. The phasic terminals (Pex) are thin and filiform in comparison. The terminals are schematically drawn from photos of a preparation stained with 4-Di-2-ASP. (C) Phasic and tonic EPSPs recorded within a single muscle fiber shows the difference in the size of the tonic and phasic responses. The first EPSPs within the train of tonic responses is usually too small to detect, but after being facilitated at $50 \mathrm{~Hz}$ the responses gradually become observable.

innervation produced large amplitude EPSPs, whereas the tonic response may not even be detected with a single stimulus. However, upon inducing facilitation with a short train of stimuli delivered at $50 \mathrm{~Hz}$ the responses became pronounced (Fig. 1C).

In examining the differential effect of 5-HT on synaptic transmission between the tonic and phasic terminals, a short train of stimuli was used for the tonic axon and a continuous $0.5 \mathrm{~Hz}$ stimulation for the phasic axon (Fig. 2). 5-HT (100 nM) had significant effects on both the tonic and phasic EPSP amplitudes (Fig. 2A, B). The amplitudes of the EPSPs within the train of events for the tonic responses and the single phasic responses were both monitored over time to ascertain a time that would be appropriate to use for comparisons among preparations. For the tonic response, the 5th, 10th, and 20th EPSPs within the train were measured. Each of these EPSPs increased equally with exposure to 5-HT. In some of the phasic preparations, a peak increase in the amplitude occurred within 1-2 min after switching the bathing medium to one containing 5-HT. This was usually followed by a slight reduction of 1-4 $\mathrm{mV}$ over the next several minutes. The enhancement in EPSP amplitudes was not observed when the medium was exchanged with saline as a sham control. Thus, the rapid rise followed by a slight reduction was only observed when the medium is changed with one containing 5-HT. Likely, this could be a rapid response of the 5-HT receptors followed either by desensitization to 5-HT or a burst of second messengers, within the terminals, which 

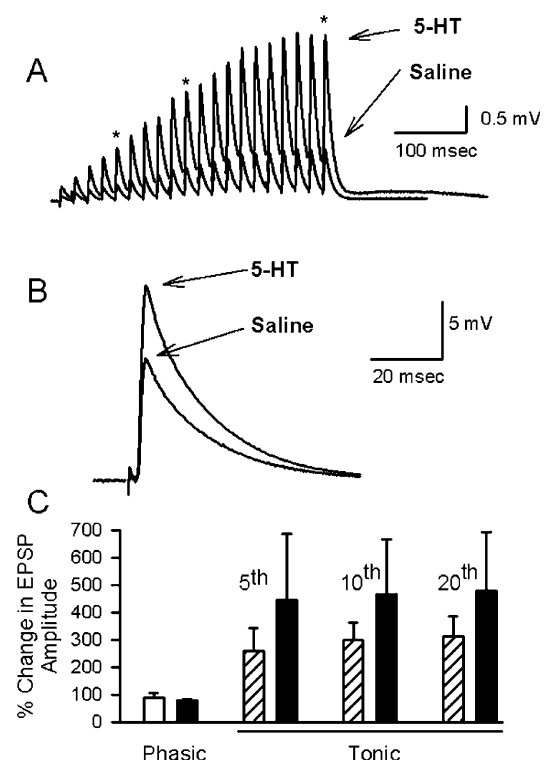

Fig. 2. Representative responses of the tonic (A) and phasic (B) EPSPs before and after exposure to 5-HT $(100 \mathrm{nM})$. The tonic axon was stimulated with a 20 pulse train delivered at $50 \mathrm{~Hz}$. Since the initial responses varied among preparations, a percent change from saline to 5-HT exposure was calculated for comparisons. A mean and standard error of the mean for 12 phasic $(n=12)$ and 5 tonic $(n=5)$ preparations are shown $(\mathrm{C})$. The open and hatched bars are of responses recorded in saline containing normal calcium concentration. The solid bars are for responses recorded in saline conditions with a $50 \%$ reduction in calcium. There is a significant effect of 5-HT enhancing phasic and tonic EPSPs $(P<0.05$, Wilcoxon rank sum test). In addition, there is a larger stimulatory effect on synaptic transmission for the tonic responses compared to the phasic ones $(P<$ 0.05 , Wilcoxon rank sum test). There is no difference in the percent change of the EPSP amplitude to 5-HT exposure between the normal and reduced calcium containing saline.

may slightly run down over time. We do not believe that the decay is due to synaptic depression since current experiments, using the same type of preparation, no synaptic depression occurs when the phasic axon is stimulated at $5 \mathrm{~Hz}$ for $15 \mathrm{~min}$ or longer (Kellie et al., 2000). To compare among preparations, a percent change of the EPSP amplitude in response to 5-HT $(100 \mathrm{nM})$ was determined for each preparation and the average ( \pm SEM) was calculated (Fig. 2C). There was an increase in all the EPSP amplitudes for both the tonic $(n=5)$ and phasic $(n=12)$ responses $(P<0.05$, Wilcoxon rank sum test) with a larger difference occurring for the tonic responses $(P<0.05$, Wilcoxon rank sum test). To test the possibility that the phasic terminals were close to saturation and thus could not produce as great of an increase in the amplitude of the EPSPs as compared to tonic responses, a saline containing 50\% reduced calcium concentration was used to dampen baseline synaptic transmission. The switch from normal saline to the reduced calcium saline resulted in the EPSPs of phasic and tonic responses to decrease in amplitude. When the reduced calcium saline bath was switched to one containing reduced calcium and 5-HT $(100 \mathrm{nM})$, the percent increase in the EPSP amplitudes for the phasic and tonic responses was not significantly different to the differences observed for 5 -Ht exposure under normal saline conditions $(n=5$ preparations for each phasic and tonic responses with reduced calcium). However, the variation was substantially larger for the tonic responses exposed to saline with reduced calcium. The increased variation suggests that the reduced calcium has a consequence in the responsiveness of the preparation to 5-HT, but the differences were not manifested in a significant change for the average EPSP amplitude. The percent change for the phasic and tonic responses to 5-HT while the preparations were exposed to reduced calcium are shown as solid bars in Fig. 2C.

\subsection{Measures of quantal currents from tonic and phasic terminals}

The number of occurrences as well as the charge of the evoked EPSCs allows one to assess the differential effects of 5-HT on the presynaptic release properties and the postsynaptic receptivity. The responses to vesicular events were monitored in the EPSC currents obtained with a focal macropatch recording electrode placed over the terminal (Fig. 3A). In the low-output tonic terminals, the evoked quantal events were directly counted for determining mean quantal content, $m$, (Fig. $3 \mathrm{~B}_{1}$, Fig. 6 and Table 1) (del Castillo and Katz, 1954; Cooper et al., 1995b). Since the phasic terminals are high-
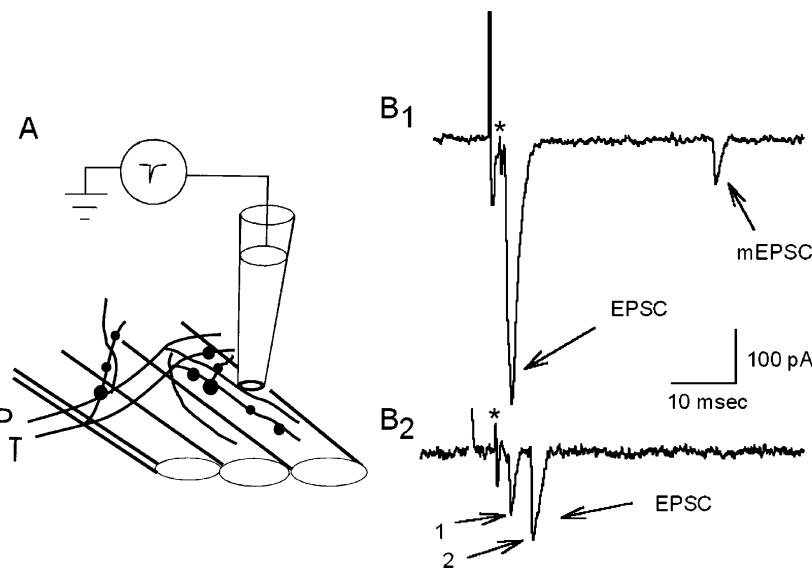

Fig. 3. EPSCs are readily recorded by placing a focal recording electrode directly over visualized regions of the tonic $(\mathrm{T})$ or phasic $(\mathrm{P})$ terminals (A). Single sweeps of evoked responses for the phasic $\left(B_{1}\right.$, top) and tonic ( $B_{2}$, bottom) terminals. The number of quantal events from an evoked stimulus are readily counted in each trace of the tonic events as a double (2) in evoking events. The direct counts are used to determine mean quantal content (Table 1). A spontaneous miniature extracellular quantal current (mEPSC) is present in one of the current traces from the phasic terminal. The extracellular nerve terminal spike is indicated with a asterisk $(*)$. The scale bars are the same for the tonic and phasic responses. 
output, the individual quantal events within the evoked EPSC are not distinguishable in physiological saline conditions (Fig. 3B). However, single spontaneous quantal events were observed (Fig. $3 \mathrm{~B}_{1}$ ) and were used, as with single evoked events measured from tonic terminals (Fig. $3 \mathrm{~B}_{2}$ ), to examine postsynaptic sensitivity. The discrete evoked quantal currents are counted as a single or multiple events as shown for the double release depicted in Fig. $3 \mathrm{~B}_{2}$ and listed in Table 1 .

\subsection{Effect of 5-HT on the synaptic charge}

The overall evoked synaptic charge in both tonic and phasic terminals rapidly increased during 5-HT exposure. A representative response to 5 -HT $(100 \mathrm{nM})$ is shown for both a phasic (Fig. 4A) and tonic (Fig. 4B) terminals. For comparison, the mean quantal content, as determined by direct quantal counts, is shown (Fig. 4C) for the same tonic responses in Fig. 4B. The response to 5-HT usually reached a plateau after a few minutes and remained constant for several more minutes. Some of the phasic preparations showed a decline in the overall charge after reaching a peak response, which is expected considering this was also observed in the EPSP recordings.

In order to quantify the effects of the 5-HT on the tonic terminals, the mean of the baseline and a segment in the plateau of the response during exposure to 5-HT
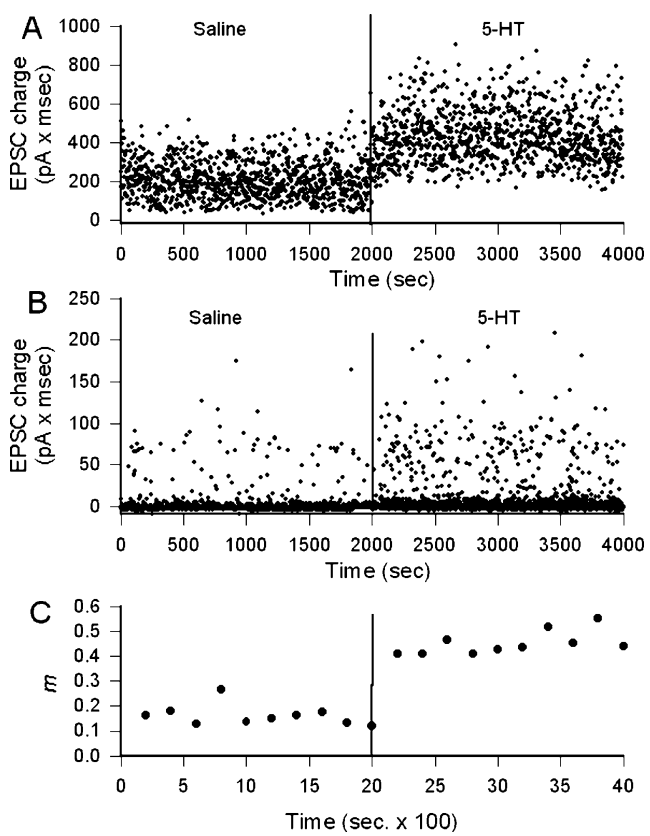

Fig. 4. Influence of 5-HT (100 nM) on synaptic currents as recorded with a focal macropatch electrode from spatially isolated regions on the phasic (A) and tonic (B) terminals. The representative scatter plots for the ESPC charge (current $\times$ time) before and during exposure to 5HT shows the rapidness of the effect. In the same preparation shown in $\mathrm{B}$ the mean quantal content was determined by direct counts of the quantal events for each 200 events and plotted over time (C). were measured and a percent change calculated. For phasic preparations, the baseline and the peak response was used. For both terminals, the differences in the synaptic charge were expressed as the percent change for comparison among preparations (Fig. 5). It is clear that the overall effect of 5-HT is to enhance synaptic transmission by causing more vesicles to be released giving rise to larger EPSCs and thus EPSPs. There appears to be little if any effect of receptivity (as measured by synaptic charge) of the postsynaptic muscle fibers, since no significant differences in the synaptic charge are detected in the distribution of spontaneous single events recorded over the tonic terminal (Fig. 6). Previous investigations on the effect of 5-HT altering input resistance of a crayfish muscle had shown a slight increase. The difference could contribute to a larger EPSP for given amount of same synaptic current (Strawn et al., 2000) but differences in the currents were not noticeable. The mean of the percent change in synaptic charge for 5 tonic and 5 phasic preparations demonstrates that the tonic terminals had a greater enhancement in synaptic transmission induced by 5 -HT as compared to phasic terminals (Fig. $5, P<0.05, n=5$ for each).

Since the majority of the effect is increasing the overall number of vesicles fusing by evoked stimulation, we further analyzed the effects of 5-HT on the release by plotting the number of failures before and during exposure to 5-HT (100 nM) (Fig. 7, Table 1). As previously reported for the tonic terminals on the opener muscle in the crayfish walking leg (Southard et al., 2000), the effect of 5-HT on the tonic motor nerve terminals in the leg extensor is an increase in the overall probability of vesicular fusion. This is shown by the increase in the number of single and multiple events presented in Table 1 for five preparations.

Since $m$ increased as a result of 5-HT exposure, a more thorough analysis in the probability of vesicular release $(p)$ and the number of release sites $(n)$ was

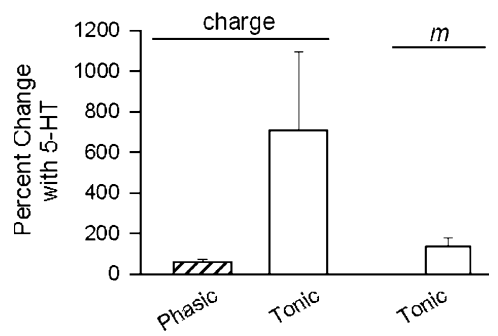

Fig. 5. The mean $( \pm$ SEM) percent change in the charge of the EPSC when exposed to 5 -HT $(100 \mathrm{nM})$ for 5 tonic and 5 phasic terminal recordings is shown. There was always an increase in the synaptic charge for each preparation ( $P<0.05$, Wilcoxon rank sum test), $n=5$ for each). Percent change is used since absolute values varied among recordings. Note there is a greater change in synaptic charge for the tonic terminals as compared to the phasic terminals. A percent change in the mean quantal content, as determined by direct counts, is also shown for the tonic preparations. 


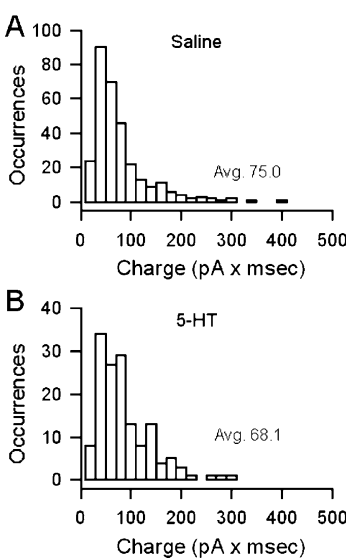

Fig. 6. The distribution in the amount of charge in the mEPSCs recorded from a tonic terminal while exposed to saline (A) and 5-HT $(100 \mathrm{nM})(\mathrm{B})$ is similar. In the particular preparation shown, the overall average in the distribution of charge is smaller during 5-HT exposure. This smaller average is due to a few giant mEPSCs recorded during saline exposure. The results support the fact that postsynaptic receptivity to glutamate is not enhanced by $5-\mathrm{HT}$.

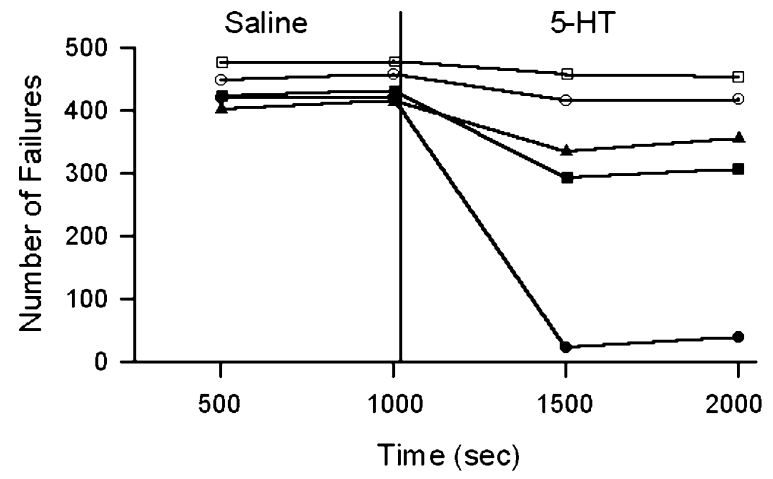

Fig. 7. The number of failures in evoked synaptic events decreases in the tonic nerve terminals after exposure to 5-HT. Groups of 500 evoked trials were compiled for indicating the various degrees of change. The nerves were stimulated at $1 \mathrm{~Hz}$. The first 1000 trials were while the nerve was exposed to saline and the second set of 1000 trials was during exposure to 5-HT. Note in all five cases shown, the number of failures is reduced when 5-HT is present.

performed (Table 1). In some cases, the observed data did not fit well to a binomial distribution but rather a Poisson distribution. With a Poisson distribution, estimation of $n$ and $p$ were not able to be determined with high accuracy. However, by definition for a Poisson distribution there is a low probability (low $p$ ) of many sites acting (high $n$ ). Since the varicosities of the tonic nerve terminals on the extensor muscle are so low in output, many of the runs at this low frequency displayed a Poisson distribution of quantal events, therefore $n$ and $p$ were not able to be estimated but simply listed as a high $n$ and a low $p$ (Table 1). Despite the inability to determine $n$ and $p$ is some cases, it is obvious in all cases that there is an increase in the number of single and multiple events occurring in the presence of 5-HT. Thus, the probability of vesicle fusion during evoked stimulation appears to be enhanced by the presence of 5-HT.

\section{Discussion}

The extensor muscle of the crayfish leg is a useful preparation for investigating differential effects of neuromodulators, like 5-HT, on synaptic transmission because the two neurons innervate the same muscle fibers. We have demonstrated that the tonic nerve terminal is more receptive to 5-HT per length of the nerve terminal as indicated by a greater increase in synaptic transmission.

It is well established that numerous neuromodulators are present in the crayfish hemolymph; but how the neuromodulators alter behavior of the whole animal are not fully understood and are somewhat controversial (Livingston et al., 1980; Yeh et al., 1996; Cooper et al., 2001; Listerman et al., 2000; Strawn et al., 2000; Doernberg et al., 2001; Tabor and Cooper, 2002). Recently it was shown that the neuromodulator octopamine in some cases antagonize 5-HT's effects of enhancing synaptic transmission (Djokaj et al., 2001). In addition, variations in responsiveness to exogenous application of neuromodulators between preparations may well be related to the prior life history of the animal. It is known that receptors to hormones or neuromodulators undergo up- and down-regulation via alteration of their expression levels and/or densities on cell surfaces (Azaryan et al., 1998) as we have also demonstrated at the crayfish NMJ (Cooper et al., 2001). This is relevant because it has been implied that the levels of 5-HT as well as other neuromodulators might be related to various social states in crustaceans (Sneddon et al., 2000).

Recent pharmacological investigations into the 5-HT receptor subtypes at the crayfish NMJ have shown some shared characteristics with the vertebrate 5-HT-2 family (Tierney and Mangiamele, 2001; Tabor and Cooper, 2002). The results are fitting for a vertebrate 5-HT-2A subfamily since earlier observations, at the crayfish NMJ, demonstrated that 5-HT induces an IP3 cascade for inducing its effects (Dixon and Atwood, 1989). As suggested by Southard et al. (2000), He et al. (1999), Cooper et al. (2001) and Tabor and Cooper (2002), 5HT could induce the phosphorylation of molecules such as Munc-18-1, synaptophysin or even NSF to enhance docking of vesicles in the absence of evoked stimulation. It is also likely that 5-HT could lead to phosphorylation of synapsins to increase the pool of free vesicles for docking (Wang and Zucker, 1998; Cooper et al., 2002) as was shown in Aplysia neurons (Angers, et al., 2002).

In considering the anatomical differences at the synaptic level of the phasic and tonic terminals, the limitation to the extent that phasic terminals can 
increase synaptic output in the presence of excitatory neuromodulators needs to be addressed. Current studies underway investigating synaptic depression of the phasic motor nerve terminals of the crayfish leg extensor muscle reveal that upon high-frequency stimulation (5 $\mathrm{Hz})$ the EPSP amplitude can initially facilitate and increase $150 \%$ in amplitude (unpublished data, Kellie et al., 2000). The mean percent increase shown herein for exposure of 5-HT (100 nM) is less than 100\% (Fig. $2 \mathrm{C})$. In addition, the experiments presented with a $50 \%$ reduced calcium in the bath showed a decrease in transmission for both the phasic and tonic responses, however no significant difference occurs in the average increase of the EPSP amplitude upon 5-HT exposure. Albeit, the variation in the percent change of the EPSP responses was considerable greater with 5-HT exposure combined with the reduced calcium concentration in the bath. Thus, the phasic terminals have not reached a maximum in their synaptic efficacy in transmission by exposure to $5-\mathrm{HT}$ at $100 \mathrm{nM}$. Since the phasic terminals are operating at a higher output, it is possible that there are not as many silent synapses that can be recruited into action by 5 -HT as for tonic terminals. In fact, it was shown that phasic terminals, in the same type of NMJ preparations as used in our study, have a 2-3 times larger amount of calcium entering during evoked stimulation as compared to tonic terminals (Msghina et al., 1999). This difference appears to be related to the differences in the calcium channels of the two terminals (Msghina et al., 1999) as well as synaptic structure in the degree of spacing between active zones on synapses (King et al., 1996; Cooper et al., 1996b; Msghina et al., 1998).

Currently, various statistical measures and derived parameters are used to quantify synaptic efficacy. Mean quantal content $(\mathrm{m})$ is commonly used as an index of the average number of events (or vesicles that fuse) that occur per stimulus. The parameter $n$ represents the number of sites that release a vesicle, whereas a third parameter, $p$, represents the probability of an event occurring at a release site (del Castillo and Katz, 1954). Since $p$ and $n$ cannot be directly measured experimentally, multiple approaches have been developed over the years to estimate $n$ and $p$ using the distribution of evoked events (see reviews-McLachlan, 1978; Faber et al., 1998). These methods have problems when synaptic efficacy is very low, in which case the observed distribution is better fit by a Poisson distribution (Zar, 1999) or possibly a binomial distribution with $n=1$. Using a Poisson distribution often results in a large estimate for $n$ and a small estimate for $p$. In other words, the release is random with a low probability but with many possible sites. In the case of the binomial distribution with $n=1$, then only one site is functional and $p$ will change as a result in differences of release. This is consistent with results in the statistical literature, in particular those of Olkin et al. (1981) indicating that simultaneous estimation of $n$ and $p$ is not stable.

The evidence to date suggest that, if possible, we should search for more information in the evoked currents that may be used to estimate $n$ and $p$. The sizes and shapes of single evoked synaptic currents is one such source. Using counting methods, two currents which both indicate one evoked event would be recorded identically even if the two currents appear distinctly different. Since differences in current sizes or shapes may indicate different sites firing, this may be useful information in determining the overall number of sites utilized. Direct structure-function studies of discrete regions of the opener tonic motor nerve terminals in crayfish have revealed that multiple sites are being utilized for vesicle fusion (Cooper et al., 1995b, 1996a,b). In addition, the standardized approach of obtaining $n$ and $p$ from methods of directly counting quantal events (del Castillo and Katz, 1954) and determining their distribution of occurrence underestimates the functional number of sites. For these reasons, we are beginning to develop novel statistical methods that incorporate differences in current sizes and shapes to estimate $n$ and $p$ (Viele et al., 2003). In the future it may be possible to revisit the data sets used in this current study to obtain better estimates of $n$ and $p$ to examine the influence of 5-HT on each parameter. Since in this study evoked release, in a number of cases, is represented by a Poisson distribution we do not feel confident to state that 5-HT alters the number of release sites, however since there is an overall increase in the number of evoked events upon 5-HT exposure, the probability of release is increased for the terminal as a whole.

Hence, there are a number of possible explanations for the differential effects of 5-HT in enhancing synaptic transmission between the phasic and tonic terminals. Firstly, it is likely that there are different densities of the 5-HT receptors on the terminals. Antibody labeling of the receptors or pharmacological ligand binding studies are needed to resolve this issue. Secondly, it is possible that there are differences in density of various sub-types of 5-HT receptors between the two terminals. Earlier, we showed that the majority of the response to 5 -HT is due to receptors within the vertebrate 5-HT-2 subfamily; but there was still a significant response that could not be blocked by antagonists to the 5-HT-2 receptor subfamily (Tabor and Cooper, 2002). Thirdly, the levels of second messengers within the terminals used to carry out cellular response of 5-HT's actions could be different. It has recently been shown, in various brain regions of the rat, that receptor numbers can stay the same but the ability to activate a 5 -HT receptor coupled G protein can be different (Hensler, 2002). Fourthly, the degree of activating silent synapses by 5-HT may be greater in the tonic terminals than the phasic terminals. Since there are 
differences in the amount of the calcium binding protein frequenin (Jeromin et al., 1999) and the amount of calcium entry (Msghina et al., 1999) between the two neurons, it seems likely that there can be a multitude of interacting differences to account for the differential effects of 5-HT. Recently in a crab, it was shown that phasic and tonic motor nerve terminals even express different types of calcium channels within the presynaptic nerve terminals. In the crab it was demonstrated by Rathmayer et al. (2002) that the two nerve terminals are regulated differently by one type of neuromodulator (i.e. DF2) but not another (i.e. proctolin). A number of more subtle issues could also come into play, but the ones mentioned are able to be readily tackled in the near future at the NMJ of this preparation.

\section{Acknowledgements}

Appreciation is given to Hye Won Cooper for illustrative work. Support was provided by a Ribble Fellowship and an Interdepartamental Neuroscience Program training fellowship, University of Kentucky, Department of Biology (J.S.) and NSF grants IBN9808631, ILI DUE-9850907 and IBN-0131459 (R.L.C.).

\section{References}

Angers, A., Fioravante, D., Chin, J., Cleary, L.J., Bean, A.J., Byrne, J.H., 2002. Serotonin stimulates phosphorylation of Aplysia synapsin and alters its subcellular distribution in sensory neurons. J. Neurosci. 22, 5412-5422.

Atwood, H.L., 1973. An attempt to account for the diversity of crustacean muscles. Am. Zool. 13, 357-378.

Atwood, H.L., 1976. Organization and synaptic physiology of crustacean neuromuscular systems. Prog. Neurobiol. 7, 291-391.

Atwood, H.L., Cooper, R.L., 1995. Functional and structural parallels in crustaceans and Drosophila neuromuscular systems. Am. Zool. $35,556-565$.

Atwood, H.L., Cooper, R.L., 1996a. Assessing ultrastructure of crustacean and insect neuromuscular junctions. J. Neurosci. Methods 69, 51-58.

Atwood, H.L., Cooper, R.L., 1996b. Synaptic diversity and differentiation: crustacean neuromuscular junctions. Invertebr. Neurosci. 1, 291-307.

Atwood, H.L., Nguyen, P.V., 1995. Neural adaptation in crayfish. Am. Zool. 35, 556-565.

Azaryan, A.V., Clock, B.J., Rosenberger, J.G., Cox, B.M., 1998. Transient up regulation of mu opioid receptor mRNA levels in nucleus accumbens during chronic cocaine administration. Can. J. Physiol. Pharmacol. 76, 278-283.

Beltz, B.S., Kravitz, E.A., 1983. Mapping of serotonin-like immunoreactivity in the lobster nervous system. J. Neurosci. 3, 585-602.

Bradacs, H., Cooper, R.L., Msghina, M., Atwood, H.L., 1997. Differential physiology and morphology of phasic and tonic motor axons in a crayfish limb extensor muscle. J. Exp. Biol. 200, 677691.

Cooper, R.L., Marin, L., Atwood, H.L., 1995a. Synaptic differentiation of a single motor neuron: conjoint definition of transmitter release, presynaptic calcium signals, and ultrastructure. J. Neurosci. $15,4209-4222$.

Cooper, R.L., Stewart, B.A., Wojtowicz, J.M., Wang, S., Atwood, H.L., 1995b. Quantal measurement and analysis methods compared for crayfish and Drosophila neuromuscular junctions and rat hippocampus. J. Neurosci. Methods 61, 67-78.

Cooper, R.L., Harrington, C., Marin, L., Atwood, H.L., 1996 a. Quantal release at visualized terminals of crayfish motor axon: intraterminal and regional differences. J. Comp. Neurol. 375, 583600.

Cooper, R.L., Winslow, J., Govind, C.K., Atwood, H.L., 1996b. Synaptic structural complexity as a factor enhancing probability of calcium-mediated transmitter release. J. Neurophysiol. 75, 24512488.

Cooper, R.L., Chase, R.J., Tabor, J., 2001. Altered responsiveness to 5-HT at the crayfish neuromuscular junction due to chronic p-CPA \& m-CPP treatment. Brain Res. 916, 143-151.

Cooper, R.L., Southard, R.C., He, P., Whiteheart, S.W., 2002. Influence of neuromodulators and vesicle docking related proteins on quantal release. In: Wiese, K. (Ed.), The Crustacean Nervous System. Springer, Heidelberg, Germany, pp. 63-82.

Crider, M.E., Cooper, R.L., 1999. The importance of the stimulation paradigm in determining facilitation and effects of neuromodulation. Brain Res. 842, 324-331.

Crider, M.E., Cooper, R.L., 2000. Differentially facilitation of highand low-output nerve terminals from a single motor neuron. J. Appl. Physiol. 88, 987-996.

del Castillo, J., Katz, B., 1954. Quantal components of the end-plate potential. J. Physiol. (Lond.) 124, 560-573.

Dixon, D., Atwood, H.L., 1989. Conjoint action of phosphoinositol and adenylate cyclase systems in serotonin-induced facilitation at the crayfish neuromuscular junction. J. Neurophysiol. 62, 12511259.

Djokaj, S., Cooper, R.L., Rathmayer, W., 2001. Effects of octopamine, serotonin, and cocktails of the two modulators on synaptic transmission at crustacean neuromuscular junctions. J. Comp. Physiol. A 187, 145-154.

Doernberg, S.B., Cromarty, S.I., Heinrich, R., Beltz, B.S., Kravitz, E.A., 2001. Agonistic behavior in naive juvenile lobsters depleted of serotonin by 5,7-dihydroxytryptamine. J. Comp. Physiol. 187A, $91-103$.

Dudel, J., 1965. Facilitatory effects of 5-hydroxy-tryptamine on the crayfish neuromuscular junction. Naunyn-Schmiedeberg's Arch.exp. Path.u. Pharmak. 249, 515-528.

Efron, B., Tibshirani, R., 1993. The bootstrap estimate of standard error. In: Efron, B., Tibshirani, R. (Eds.), An Introduction to the Bootstrap. Chapman Hall, pp. 45-59.

Faber, D.S., Korn, H., Redman, S.J., Thompson, S.M., Altman, J.S., 1998. Central Synapses: Quantal Mechanisms and Plasticity. Human Frontier Science Program, Strasbourg.

Fisher, L., Florey, E., 1983. Modulation of synaptic transmission and excitation-contraction coupling in the opener muscle of the crayfish, Astacus leptodactylus, by 5-hydroxytryptamine and octopamine. J. Exp. Biol. 102, 187-198.

Florey, E., Cahill, M.A., 1982. The innervation pattern of crustacean skeletal muscle. Cell Tissue Res. 224, 527-541.

Florey, R.E., Florey, E., 1954. Über die mögliche Bedeutung von Enteramin (5-Oxy-Tryptamine) als nervöser Aktionssubstanz bei Cephalopoden und dekapoden Crustaceen. Z. Naturforsch. 9b, $58-69$.

He, P., Southard, R.C., Whiteheart, S.W., Cooper, R.L., 1999. Role of $\alpha$-SNAP in promoting efficient neurotransmission at the crayfish neuromuscular junction. J. Neurophysiol. 82, 3406-3416.

Hensler, J.G., 2002. Differential regulation of 5- $\mathrm{HT}_{1 \mathrm{~A}}$ receptor-G protein interactions in brain following chronic antidepressant administration. Neuropsychopharmacology 26, 565-573. 
Jeromin, A., Shayan, A.J., Msghina, M., Roder, J., Atwood, H.L., 1999. Crustacean frequenins: molecular cloning and differential localization at neuromuscular junctions. J. Neurobiol. 41, 165175.

Kellie, S.P., Bradacs, H., Cooper, R.L., 2000. Mechanisms of synaptic depression in high output phasic motor neurons. Abst. Soc. Neurosci. 26, 518.10.

King, M.J.R., Atwood, H.L., Govind, C.K., 1996. Structural features of crayfish phasic and tonic neuromuscular junctions. J. Comp. Neurol. 372, 618-626.

Kupfermann, I., 1979. Modulatory actions of neurotransmitters. Ann. Rev. Neurosci. 2, 447-465.

LaFramboise, W., Griffis, B., Bonner, P., Warren, W., Scalise, D., Guthrie, R.D., Cooper, R.L., 1999. Muscle type-specific myosin isoforms in crustacean muscles. J. Exp. Zool. 286, 36-48.

Listerman, L., Deskins, J., Bradacs, H., Cooper, R.L., 2000. Measures of heart rate during social interactions in crayfish and effects of 5HT. Comp. Biochem. Physiol. A 125, 251-264.

Livingston, M.S., Harris-Warrick, R.M., Kravitz, E.A., 1980. Serotonin and octopamine produce opposite postures in lobsters. Science 208, 76-79.

Magrassi, L., Purves, D., Lichtman, J.W., 1987. Fluorescent probes that stain living nerve terminals. J. Neurosci. 7, 1207-1214.

McLachlan, E.M., 1978. The statistics of transmitter release at chemical synapses. In: Porter, R. (Ed.), International Review of Physiology, Neurophysiology III, vol. 17. University Park Press, Baltimore, pp. 49-117.

Msghina, M., Govind, C.K., Atwood, H.L., 1998. Synaptic structure and transmitter release in crustacean phasic and tonic motor neurons. J. Neurosci. 18, 1374-1382.

Msghina, M., Millar, A.G., Charlton, M.P., Govind, C.K., Atwood, H.L., 1999. Calcium entry related to active zones and differences in transmitter release at phasic and tonic synapses. J. Neurosci. 19, 8419-8434.

Olkin, I., Petkau, A.J., Zidek, J.V., 1981. A comparison of $n$ estimators for the binomial distribution. J. Am. Stat. Assoc. 76, 637-642.

Quigley, P.A., Msghina, M., Govind, C.K., Atwood, H.L., 1999. Visible evidence for differences in synaptic effectiveness with activity-dependent vesicular uptake and release of FM1-43. J. Neurophysiol. 81, 356-370.
Rathmayer, W., Djokaj, S., Gaydukov, A., Kreissl, S., 2002. The neuromuscular junctions of the slow and fast excitatory axon in the closer of the crab Eriphia spinifrons are endowed with different $\mathrm{Ca}^{2+}$ channel types and allow neuron-specific modulation of transmitter release by two neuropeptides. J. Neurosci. 22, 708-717.

Shearer, J., Zolman, J.F., Cooper, R.L., 2000. The differential effects of 5-HT on tonic and phasic motor nerve terminals. Abst. Soc. Neurosci. 26, 441.17.

Smith, B.R., Wojtowicz, J.M., Atwood, H.L., 1991. Maximum likelihood estimation of non-uniform transmitter release probabilities at the crayfish neuromuscular junction. J. Theor. Biol. 150, 457472 .

Sneddon, L.U., Taylor, A.C., Huntingford, F.A., Watson, D.G., 2000. Agonistic behavior and biogenic amines in shore crabs Carcinus maenas. J. Exp. Biol. 203, 537-545.

Southard, R.C., Haggard, J., Crider, M.E., Whiteheart, S.W., Cooper, R.L., 2000. Influence of serotonin on the kinetics of vesicular release. Brain Res. 871, 16-28.

Strawn, J.R., Neckameyer, W.S., Cooper, R.L., 2000. The effects of 5HT on sensory neurons, CNS command, and neuromuscular junctions of central and motor neurons driving the crayfish abdominal superficial flexor muscle in the crayfish. Comp. Biochem. Physiol. B 127, 544-550.

Tabor, J., Cooper, R.L., 2002. Physiologically identified 5-HT 2 -like receptors at the crayfish neuromuscular junction. Brain Res. 932, $87-94$.

Tierney, A.J., Mangiamele, L.A., 2001. Effects of serotonin and serotonin analogs on posture and agonistic behavior in crayfish. J. Comp. Physiol. A 187, 757-767.

Viele, K., Stromberg, A., Cooper, R.L., 2003. Estimating the number of release sites and probability of firing within the nerve terminal by statistical analysis of synaptic charge. Synapse 47, 15-25.

Wang, C., Zucker, R.S., 1998. Regulation of synaptic vesicle recycling by calcium and serotonin. Neuron 21, 155-167.

Wojtowicz, J.M., Smith, B.R., Atwood, H.L., 1991. Activity-dependent recruitment of silent synapses. Ann. N.Y. Acad. Sci. 627, $169-179$.

Yeh, S.R., Fricke, R.A., Edwards, D.H., 1996. The effect of social experience on serotonergic modulation of the escape circuit of crayfish. Science 271, 366-369.

Zar, J.H., 1999. Biostatistical Analysis. Prentice Hall, New Jersey. 